\title{
Size-Dependent Reactivity in Hydrosilylation of
}

\section{Silicon Nanocrystals}

Joel A. Kelly, ${ }^{a}$ Amber M. Shukaliak, ${ }^{a}$ Michael D. Fleischauer, ${ }^{b}$ Jonathan G.C. Veinot ${ }^{a,{ }^{*}}$

${ }^{a}$ Department of Chemistry, University of Alberta, Edmonton Alberta T6G 2G2, Canada

${ }^{\mathrm{b}}$ NRC- National Institute for Nanotechnology, Edmonton Alberta T6G 2M9, Canada

Corresponding author: jveinot@ualberta.ca, Fax: 780-492-8231. Tel.: 780-492-7206

\section{Supporting Information}

\section{Small Angle X-ray Scattering Theory and Fitting}

The scattering intensity $(I(q))$ of a dilute dispersion of scatterers is given by equation $1:^{1}$

$$
I(q) \propto \int_{0}^{\infty} N(R) P(q R) R^{6} d R
$$

where $P(q R)$ is the form factor; for a dispersion of solid homogeneous spheres this is described by equation 2 :

$$
P(q R)=9\left(\frac{\sin (q R)-q R \cos (q R)}{(q R)^{3}}\right)^{2}
$$


$q$ is the scattering wave vector, related to the scattering angle and X-ray wavelength by equation 3:

$$
q=\frac{4 \pi}{\lambda} \sin \left(\frac{\theta}{2}\right)
$$

The number fraction of scatters of radius $R, N(R)$, was assumed to follow a Gaussian distribution, given by equation 4 :

$$
N(R)=\sigma \sqrt{(2 \pi)} e^{\frac{(-R-\bar{R})^{2}}{2 \sigma^{2}}}
$$

where $\bar{R}$ is the mean nanocrystal radius and $\sigma$ is the standard deviation.

Least squares fitting using the parameters $\bar{R}$ and $\sigma$ was carried out on the "small" and "large" Si-NC SAXS data using the Irena package for Igor Pro. "Combined" samples were fit by fixing the parameters $\bar{R}$ and $\sigma$ obtained for each separate population and allowing the volume fraction of each population to vary.

To verify this fitting approach for the "combined" samples as an accurate determination of the relative volume fraction of each population, the "small" and "large" silicon nanocrystal (Si-NC) samples were mixed in known ratios (i.e., 1:2, 1:1, 10:1 and 25:1 "small": "large") and modeled using SAXS as described above (Figure S1). 


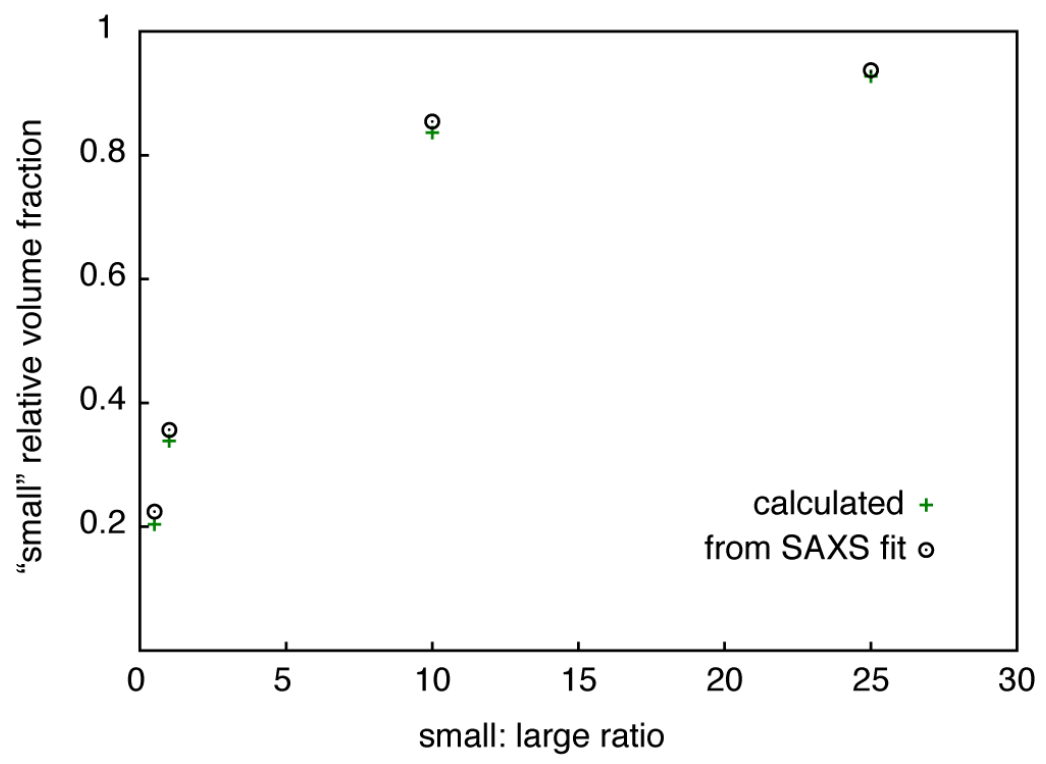

Figure S1: SAXS calibration curve of "small" and "large" samples mixed in known ratios.

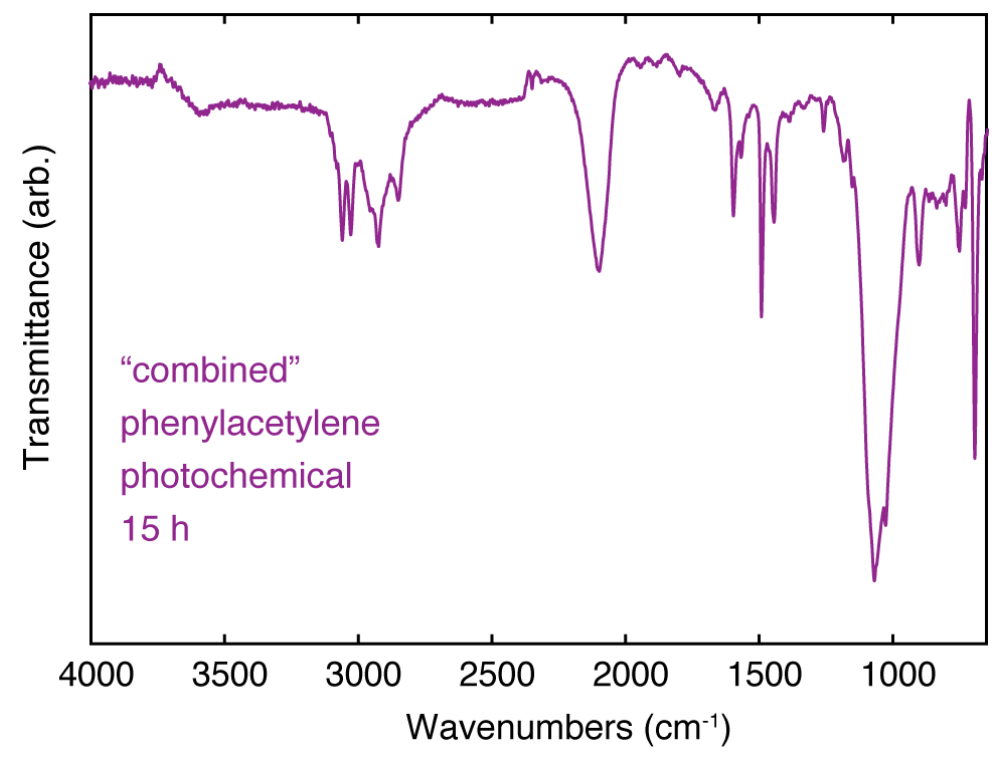

Figure S2: FTIR of the "combined" Si-NC sample photochemically functionalized with phenylacetylene. 


\section{References}

(1) Small-angle X-ray Scattering; Glatter, O; Kratky, O., Eds.; Academic Press: New York, 1982.

(2) Ilavsky, J.; Jemian, P. R. J. Appl. Crystalogr. 2009, 42, 347-353. 